\title{
Clear cell carcinoma of the lower uterine segment: A case report
}

\author{
YUSUKE MATOBA $^{1,2}$, IORI KISU ${ }^{1,2}$, KEIKO SAOTOME $^{1}$, MOTOKO KATAYAMA ${ }^{1}$, \\ MAKIKO TANIGUCHI $^{1}$, YUMIKO MIURA ${ }^{1}$, TAEKO GOTO ${ }^{1}$ and NOBUMARU HIRAO ${ }^{1}$ \\ ${ }^{1}$ Department of Obstetrics and Gynecology, Federation of National Public Service Personnel Mutual Aid Associations, \\ Tachikawa Hospital, Tokyo 190-0022; ${ }^{2}$ Department of Obstetrics and Gynecology, \\ Keio University School of Medicine, Keio University, Tokyo 160-8582, Japan
}

Received January 13, 2016; Accepted September 26, 2016

DOI: $10.3892 / \mathrm{mco} .2016 .1048$

\begin{abstract}
Uterine carcinoma of the lower uterine segment (LUS) is a rare tumor that accounts for $3-3.5 \%$ of cases of uterine malignant cancer. The tumor arises from the lower region of the uterine body through the upper region of the cervix. The present study reported a case of clear cell carcinoma that originated from the LUS. A 50-year-old woman visited a local hospital due to irregular vaginal bleeding. She was suspected to have a uterine tumor and was referred to Tachikawa Hospital (Tokyo, Japan). Transvaginal ultrasound and magnetic resonance imaging revealed a uterine tumor from the lower region of the uterine body through the upper region of the cervix. Endocervical curettage revealed clear cell carcinoma. Based on a diagnosis of clear cell carcinoma of the LUS, radical hysterectomy was performed with bilateral salpingo-oophorectomy, paraaortic lymph node dissection and omentectomy. Macroscopically, the tumor was limited to the lower region of the uterine body through the upper region of the cervix in the resected uterus. Histopathological findings indicated no tumors in the uterine corpus and uterine cervix, but clear cell carcinoma was observed in the LUS epithelium. At the 1-year follow-up, the patient remained free of local recurrence and metastasis. To the best of our knowledge, clear cell carcinoma of the LUS has not previously been reported. More cases are required to clarify the pathology.
\end{abstract}

\section{Introduction}

Carcinoma of the lower uterine segment (LUS) is a malignant uterine tumor arising between the anatomical internal os and histological internal os of the uterus. It is a rare tumor that accounts for $3-3.5 \%$ of uterine corpus carcinomas (1).

Correspondence to: Dr Iori Kisu, Department of Obstetrics and Gynecology, Keio University School of Medicine, Keio University, 35 Shinanomachi, Shinjuku-ku, Tokyo 160-8582, Japan

E-mail: iori71march@hotmail.co.jp

Key words: lower uterine segment, clear cell carcinoma, isthmus, Lynch syndrome, endometrial carcinoma
Carcinoma of the LUS is characterized by deeper myometrial invasion and multiple lymph node metastases compared with endometrial carcinoma, and a previous study indicated an association with Lynch syndrome (1). A cancer that is widely present in the whole body through the endocervix is excluded from the definition of carcinoma of the LUS. It is often difficult to distinguish between cervical cancer and uterine corpus cancer; however, the treatment policies are different. Magnetic resonance imaging (MRI) may be able to discriminate between the two conditions (2); other reports suggest that MRI is not necessarily useful in this respect (3). Discrimination may also be possible by immunohistochemical staining (4) or detection of human papillomavirus (HPV) DNA (5); however, there is no established method for this purpose.

Clear cell carcinoma of the uterine corpus is a rare tumor that accounts for $1-6 \%$ of uterine corpus carcinomas. Clear cell carcinoma is considered to be a type II tumor that is less associated with estrogen and differs from endometrioid adenocarcinoma (1). Following surgical staging, patients with clear cell carcinoma are often upstaged to a higher clinical stage compared with that found in pre-surgical staging. Clear cell carcinoma is also less sensitive to chemotherapy and radiotherapy compared with lower grade endometrioid adenocarcinoma (6). Therefore, the 5-year overall survival rate of clear cell carcinoma of the uterine corpus is only $\sim 40 \%$, which is markedly worse compared with that of endometrioid adenocarcinoma (6). The present report described a rare case of clear cell carcinoma arising from the LUS and is presented with written informed consent from the patient.

\section{Case report}

The patient was a 50-year-old woman (gravida 2, para 2) with no other relevant medical and family history. She visited a local clinic due to irregular vaginal bleeding for several months. She was diagnosed with a uterine tumor and referred to Tachikawa Hospital (Tokyo, Japan). Transvaginal ultrasound revealed a uterine tumor of $\sim 3 \mathrm{~cm}$ from the lower region of the uterine body through the upper region of the cervix (Fig. 1). Serum carbohydrate antigen (CA)-125 was elevated to $111.0 \mathrm{U} / \mathrm{ml}$ (normal, <35 U/ml). Other tumor markers (SCC, CA19-9 and CEA) were within normal ranges and other biochemical parameters in the blood were normal. MRI revealed the presence of a tumor mass protruding into the uterine cavity from the lower 
portion of the uterine body to the upper uterine cervix (Fig. 2). A cervical pap smear was negative for intraepithelial lesions. An endocervical smear revealed adenocarcinoma. A curettage biopsy of the uterine cervix revealed atypical cells with atypical glands, and papillary formations and clear cytoplasm, leading to diagnosis of clear cell carcinoma. An endometrial biopsy was negative. Computed tomography revealed no obvious lymph node metastasis or distant metastasis.

Based on a preoperative diagnosis of clear cell carcinoma of the LUS, radical hysterectomy was performed with bilateral salpingo-oophorectomy, pelvic lymph node dissection, paraaortic lymph node dissection and omentectomy. Macroscopically, a tumor of $4.0 \times 5.5 \mathrm{~cm}$ was observed focally in the LUS, with no tumors in the uterine corpus and cervix (Fig. 3). No abnormal histopathological findings were observed in the endometrium and uterine cervix epithelium, but malignant cells with well-defined borders with the uterine cervix epithelium and endometrial epithelium (front formation) was observed in the LUS (Fig. 4A and B). The malignant cells exhibited tubular and papillary formations. Additionally, hobnail cells with large nuclei, chromatin condensation and high-grade dyskaryosis, and abundant clear cytoplasms were observed. Based on these findings, the tumor was diagnosed as clear cell carcinoma (Fig. 4C). Cervical stromal invasion was marginally identified; however, no metastasis into the adnexa or lymph nodes was observed. Finally, the tumor was diagnosed as endometrial clear cell carcinoma arising from the LUS (FIGO stage II). Adjuvant chemotherapy was performed. At the 1-year follow-up, the patient was disease-free without local recurrence or metastasis.

\section{Discussion}

Carcinoma of the LUS is managed as a cancer of the uterine corpus; however, the characteristics differ from those of other cancer types of the uterine corpus. The age of onset of carcinoma of the LUS is younger $(3,7)$ and the tumor lacks type I characteristics, which include irregular menstruation, nulliparity, infertility and a high frequency of polycystic ovary syndrome (7). Carcinoma of the LUS has also recently been associated with Lynch syndrome. Westin et al (3) found that 10/35 cases (29\%) of carcinoma of the LUS also had Lynch syndrome and that the hMSH2 mutation was present at a high frequency. Hachisuga et al (7) reported that carcinoma of the LUS was more frequently adenosquamous carcinoma with grade 3 pathological features (7), whereas Watanabe et al (8) and Westin et al (3) suggested that no difference in the histological type and grading existed between carcinoma of the LUS and cancer of the uterine corpus. Deeper muscular invasion of carcinoma of the LUS was observed compared with that of cancer of the uterine corpus $(3,7)$. In addition, the frequency of metastasis-positive lymph node cytology in ascites, lymph and blood vessels is higher in carcinoma of the LUS $(3,8)$. These characteristics are suggested to be due to the endometrium of the LUS being thinner compared with that of the uterine corpus.

It is often difficult to determine whether a tumor originates from the LUS or cervix, though the treatment differs for the different tumor types. Haider et al (2) found that MRI is useful for discriminating between endometrial cancer

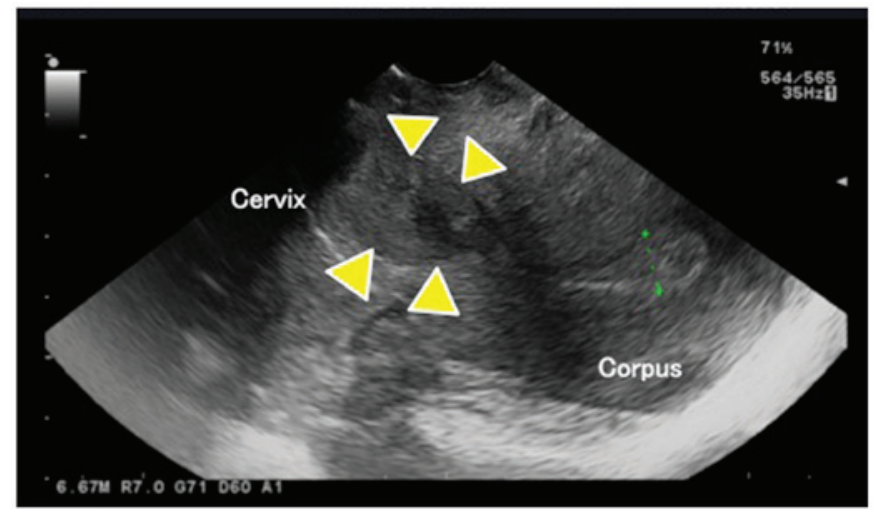

Figure 1. Transvaginal ultrasound findings. A transvaginal ultrasound revealed a uterine tumor of $\sim 3 \mathrm{~cm}$ that was present from the lower region of the uterine body through to the upper region of the cervix (yellow triangle).

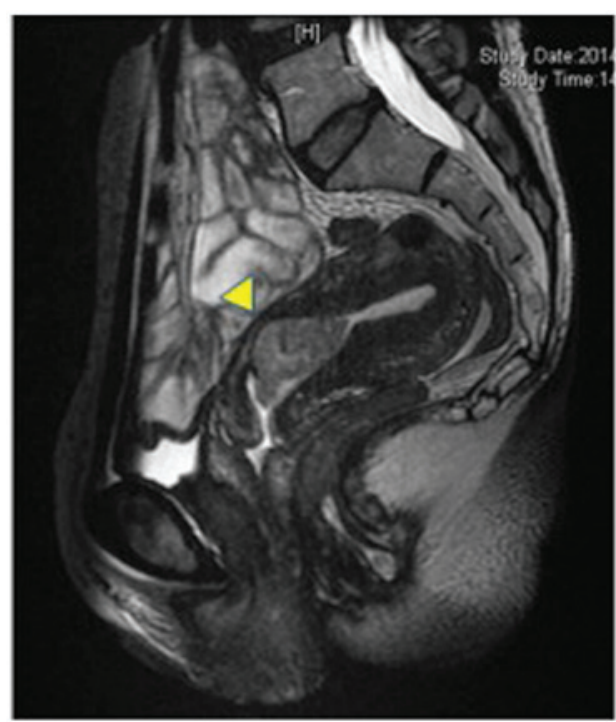

Figure 2. T2-weighted sagittal MRI findings. T2-weighted sagittal MRI revealed the presence of a tumor mass protruding into the uterine cavity from the lower portion of the uterine body to the upper uterine cervix (yellow triangle). MRI, magnetic resonance imaging.

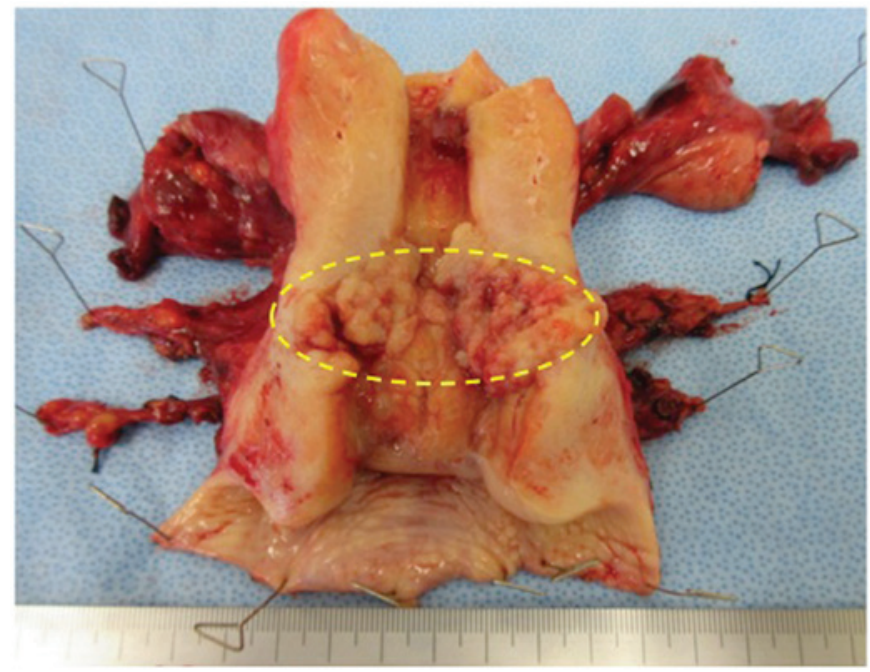

Figure 3. Macroscopic findings. Macroscopic examination of the resected uterus revealed a tumor of $4.0 \times 5.5 \mathrm{~cm}$, that was limited to the lower uterine segment (yellow circle), with no tumors in the uterine corpus and cervix. 
with cervical invasion and cervical cancer, with a positive predictive value of $92 \%$ and a negative predictive value of $88 \%$ for discriminating between these conditions (2). By contrast, Westin et al (3) found that MRI was not necessarily useful for discrimination based on the inability to diagnose the cancer origin in $56 \%$ of cases with uncertain primary lesions, in which $23 \%$ of patients originally diagnosed with cervical cancer were finally diagnosed with cancer of the LUS (3). Immunohistochemical analysis is another approach for discrimination. Typical cases of endometrial cancer are positive for estrogen receptor (ER) and vimentin, and negative for carcinoembryonic antigen (CEA), whereas cervical cancer exhibits the opposite pattern. Therefore, it has been suggested that a combination of markers may allow discrimination of cervical cancer from endometrial cancer $(4,9)$. Detection of HPV DNA and immunostaining for p16 may also be useful for discrimination of these conditions (5).

In the present case, discrimination was relatively easy preoperatively since the tumor was limited to the LUS. In general, pathological features of both the uterine endocervix and endometrium are observed in the LUS epithelium and interstitial tissue. However, the pathological findings in isolated specimens in this case revealed that epithelia of the uterine cervix and corpus were normal, and identified malignant cells with well-defined borders within these epithelia. Clear cell carcinoma originating from the LUS was definitively diagnosed histopathologically.

Clear cell carcinoma exhibits different characteristics compared with endometrial cancer. Clear cell carcinoma of the uterine corpus accounts for $1-6 \%$ of uterine corpus carcinomas (6), and is considered to be a poorly differentiated carcinoma, such as serous adenocarcinoma. These tumors are classified as type II uterine corpus cancer and are less associated with estrogen. Clear cell carcinoma has a high propensity toward extrauterine spread and a poor prognosis, with a previous study finding that $39 \%$ of patients with clinical stage I or II clear cell carcinoma were upstaged to III or IV, compared with $12 \%$ with an endometrioid subtype (6). Surgical treatment and adjuvant therapy are used; however, there is limited evidence for the efficacy of chemotherapy and radiation therapy.

To the best of our knowledge, this is the first report of clear cell carcinoma of the LUS. The tumor exhibited the characteristics of cancer of the LUS and of clear cell carcinoma. A search for 'clear cell' and 'lower uterine segment' on Pubmed resulted in no reports of clear cell carcinoma of the LUS. Carcinoma of the LUS itself is relatively rare, and therefore, a tumor of the LUS with clear cell carcinoma is particularly unusual. Cancer of the LUS and uterine clear cell carcinoma exhibit characteristics of type II uterine corpus cancer and may have common risk factors. The present patient had delivered two children and did not exhibit irregular menstruation or other risk factors for type I uterine corpus cancer. This background is consistent with the characteristics of cancer of the LUS; however, muscle invasion only at the endocervix, a negative cytological examination in ascitic fluid, lack of vascular invasion, and negative lymph node metastasis are inconsistent with a tumor of the LUS. Therefore, more cases are required to clarify the pathology of clear cell carcinoma of the LUS.
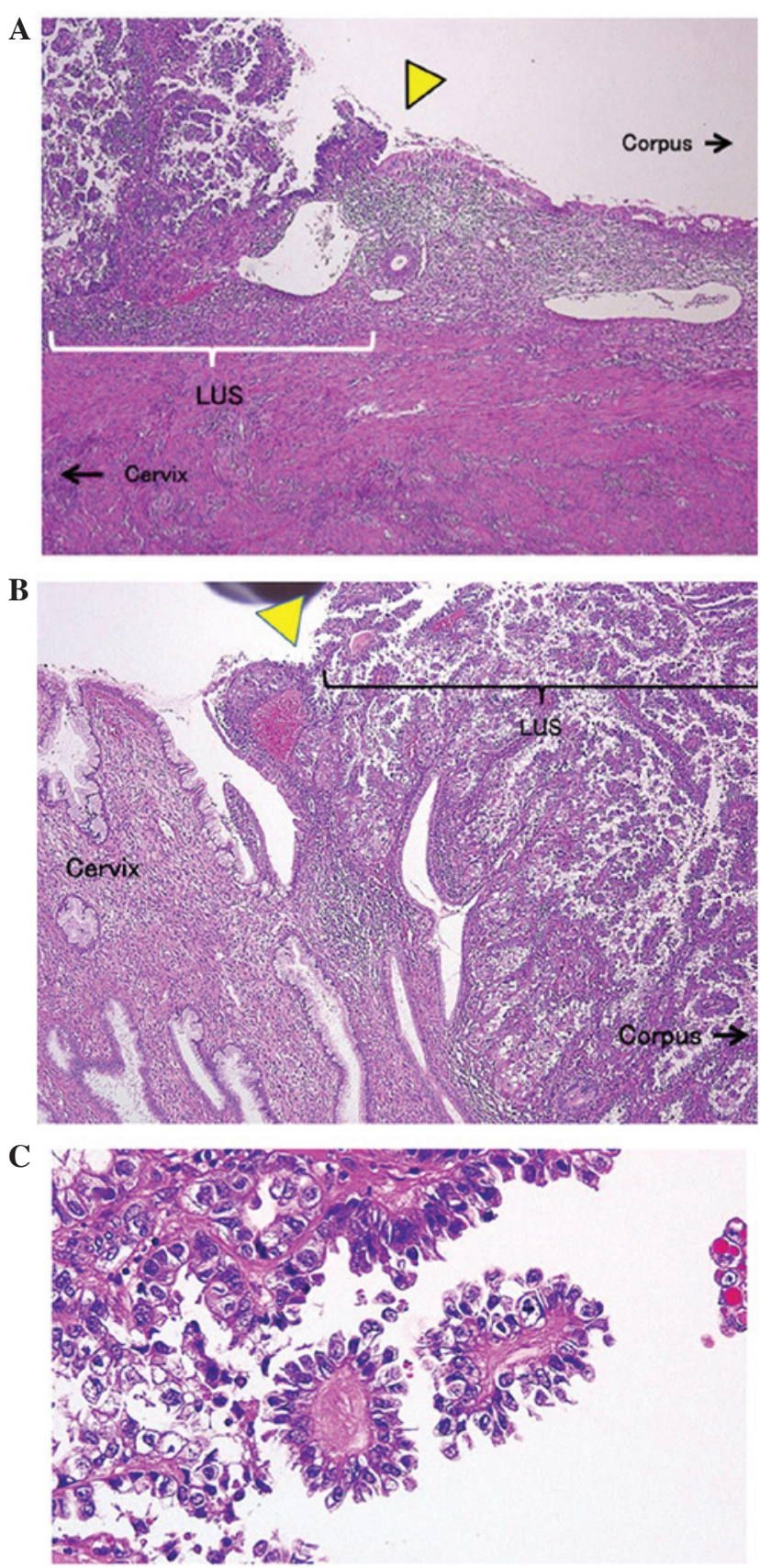

Figure 4. Pathological findings for the LUS. (A) Malignant cells with solid and papillary proliferation of the endometrium in the LUS. The epithelium of the uterine corpus was normal. Tumor cells originating from the LUS epithelium formed a border with the endometrial epithelium (yellow triangle) (magnification, $\mathrm{x} 4$ ). (B) The epithelium of the uterine cervix was normal. Tumor cells originating from the LUS epithelium formed a border (front formation) with the epithelium of the uterine cervix (yellow triangle) (magnification, x20). (C) Clear cell carcinoma containing hobnail cells with high-grade dyskaryosis and abundant clear cytoplasms (magnification, x20). LUS, lower uterine segment.

\section{References}

1. Masuda K, Banno K, Yanokura M, Kobayashi Y, Kisu I, Ueki A, Ono A, Nomura H, Hirasawa A, Susumu N, et al: Carcinoma of the lower uterine segment (LUS): Clinicopathological characteristics and association with Lynch Syndrome. Curr Genomics 12: 25-29, 2011.

2. Haider MA, Patlas M, Jhaveri K, Chapman W, Fyles A and Rosen B: Adenocarcinoma involving the uterine cervix: Magnetic resonance imaging findings in tumours of endometrial, compared with cervical, origin. Can Assoc Radiol J 57: 43-48, 2006. 
3. Westin SN, Lacour RA, Urbauer DL, Luthra R, Bodurka DC, $\mathrm{Lu} \mathrm{KH}$ and Broaddus RR: Carcinoma of the lower uterine segment: A newly described association with Lynch syndrome. J Clin Oncol 26: 5965-5971, 2008.

4. McCluggage WG, Sumathi VP, McBride HA and Patterson A: A panel of immunohistochemical stains, including carcinoembryonic antigen, vimentin, and estrogen receptor, aids the distinction between primary endometrial and endocervical adenocarcinomas. Int J Gynecol Pathol 21: 11-15, 2002.

5. Ansari-Lari MA, Staebler A,Zaino RJ, Shah KV and Ronnett BM: Distinction of endocervical and endometrial adenocarcinomas: Immunohistochemical p16 expression correlated with human papillomavirus (HPV) DNA detection. Am J Surg Pathol 28: 160-167, 2004

6. Hasegawa K, Nagao S, Yasuda M, Millan D, Viswanathan AN, Glasspool RM, Devouassoux-Shisheboran M, Covens A, Lorusso D, Kurzeder C, et al: Gynecologic Cancer InterGroup (GCIG) consensus review for clear cell carcinoma of the uterine corpus and cervix. Int J Gynecol Cancer 24 (Suppl 3): S90-S95, 2014.
7. Hachisuga T,Fukuda K, Iwasaka T,Hirakawa T,Kawarabayashi T and Tsuneyoshi M: Endometrioid adenocarcinomas of the uterine corpus in women younger than 50 years of age can be divided into two distinct clinical and pathologic entities based on anatomic location. Cancer 92: 2578-2584, 2001.

8. Watanabe Y, Nakajima H, Nozaki K, Ueda H, Obata K, Hoshiai H and Noda K: Clinicopathologic and immunohistochemical features and microsatellite status of endometrial cancer of the uterine isthmus. Int J Gynecol Pathol 20: 368-373, 2001

9. Liao CL, Hsu JD, Lee MY, Kok LF, Li YJ, Wang PH, Yao CC and Han CP: Distinguishing between primary endocervical and endometrial adenocarcinomas: Is a 2-marker (Vim/CEA) panel enough? Virchows Arch 456: 377-386, 2010. 